\title{
THE CHEBYSHEV APPROXIMATION METHOD*
}

\author{
BY \\ PRESTON R. CLEMLNT** \\ Electrical Engincering Department, Princeton C'niwer.it!
}

A treatment of the general (hehysher approximation method as it interests physicists and engineers is given, with a detailed discussion of the properties of (hebysher polynomials. Brief applications to electric circuit theory are presented.***

Introduction. Of the various means of approximating a given function, the Chebyshev method is, for physicists and engineers, one of the most interesting and important. Originally introduced by P. I. ('hebyshev in a mechanical linkage problem, (26) this procedure came into particular importance in electrical engineering with the publication of a new method of filter design by $\mathrm{W}$. Cauer.(5)

It is the aim of this paper to review the various articles written on this subject, with the purpose of presenting the material in a manner sufficiently general to permit physicists and engineers to appreciate fully this approximation problem. Particular emphasis is given to Chebyshev polynomials, with brief applications to electric circuit theory.

1. The Problem of Approximation. Chebyshev Approximation. Consider a function $f(x)$ defined in an interval $a \leqq x \leqq b$. Let $g_{n}\left(x ; a_{i}\right)$ be a function defined in the same interval with $n$ adjustable parameters $a_{1}, \cdots, a_{n}$. Our problem is the following: What should be the values of the $n$ parameters in order that $g_{n}\left(x ; a_{i}\right)$ will approximate $f(x)$ as well as possible in the interval $(a, b)$ ?

The answer to this question depends, of course, on what is meant by the expression "as well as possible." The applied mathematician is interested in obtaining a function $g_{n}\left(x ; a_{i}\right)$ which will be easier to use in his calculations than the original function $f(x)$, yet which will produce a result sufficiently close to the exact answer. A frequently used approximation is that of least squares; that is, the $a$ 's are adjusted so that

$$
\int_{a}^{b}\left[f(x)-g_{n}\left(x ; a_{i}\right)\right]^{2} d x
$$

is a minimum. For example, if the interval $(a, b)$ is $(-\pi, \pi)$, if $f(x)$ is odd about the origin, and if $g_{n}\left(x ; a_{i}\right)=a_{1} \sin x+a_{2} \sin 2 x+\cdots+a_{n} \sin n x$, then the least squares approximation will produce the Fourier coefficients for the $a$ 's.

Let us now consider an engineering problem, the design of filter networks on an amplitude spectrum basis. Suppose that it is desired to have a transmission characteristic $f(x)$ which has a constant non-zero value in the pass bands and a zero value everywhere else. It is clear that with a physically realizable circuit such a characteristic can only be approximated. Thus in this case, we can denote by $g_{n}\left(x ; a_{i}\right)$ the amplitude spectrum of the actual filter circuit which should approximate our ideal characteristic $f(x)$ "as well as possible." $x$ will denote the frequency, and the $a_{i}$ will be numbers determined by the adjustable circuit parameters of the system.

For the purpose of filtering, it is clear that the least squares approximation may not

*Received July 18, 1952.

**Now on military leave.

***This research was supported in part by funds of the Eugene Higgins Trust allocated to Princeton University. 
be a very satisfactory one, since one may desire the guarantee that in the stopbands the attenuation for every frequency is above a certain definite value. With the least squares approximation, the attenuation in the mean of a frequency mixture in the stopbands is indeed very high, but for one or more frequencies it may be quite small.

Thus to insure that such large departures from the ideal characteristic do not occur for any frequency, we would demand that the maximum of

$$
\left|f(x)-g_{n}\left(x ; a_{i}\right)\right|
$$

be a minimum in the prescribed interval $(a, b)$. When such is the case, $g_{n}\left(x ; a_{1}\right)$ is said to approximate $f(x)$ in the Chebyshev sense in the interval $(a, b)$. Hereafter, we will mean by "approximate," approximate in the Chebyshev sense.

If we let $h_{n}\left(x ; a_{i}\right)=f(x)-g_{n}\left(x ; a_{i}\right)$, our general problem becomes the following:

Given an arbitrary function $h_{n}\left(x ; a_{i}\right)$ with $n$ parameters $a_{1}, \cdots, a_{n}$, what should be the values of these parameters to make the maximum difference from zero of $h_{n}\left(x ; a_{i}\right)$ a minimum for $a \leqq x \leqq b$; i.e., so that $h_{n}\left(x ; a_{i}\right)$ approximates zero in $(a, b)$ ?

2. The Function $h_{n}\left(x ; a_{i}\right)$. In all that follows in this section, it will be assumed that $h_{n}\left(x ; a_{i}\right)$ is an arbitrary function defined in the interval $a \leqq x \leqq b$, subject to the restriction that the function and its derivatives with respect to $x, a_{1}, \cdots, a_{n}$ are finite and continuous. In order to determine the values of the parameters for the maximum deviation of $h_{n}\left(x ; a_{i}\right)$ from zero to be a minimum, the actual value of this deviation, and the points at which this deviation is assumed, two sets of equations will be considered.

The first set arises simply by noting that if $L_{n}$ is the smallest possible maximum deviation of $h_{n}\left(x ; a_{i}\right)$ from zero in $(a, b)$, then when the parameters are properly adjusted, the equation

$$
h_{n}^{2}\left(x ; a_{i}\right)-L_{n}^{2}=0
$$

will give the set of points $x_{1}, x_{2}, \cdots, x_{\mu}$ in $(a, b)$ at which the maximum is assumed. If the value $L_{n}$ is assumed on the boundary of the interval, then two of the points of the set $x_{1}, \cdots, x_{\mu}$ are $a$ and $b$. The rest of the points are on the interior, where the first derivative must vanish. Hence if differentiation with respect to $x$ is denoted by a prime, the first set of equations to be satisfied is

$$
\left.\begin{array}{r}
h_{n}^{2}\left(x ; a_{i}\right)-L_{n}^{2}=0 \\
(x-a)(x-b) h_{n}^{\prime}\left(x ; a_{i}\right)=0 .
\end{array}\right\}
$$

These equations will have $\mu$ common solutions which are real and distinct; $x_{1}, x_{2}, \cdots$, $x_{\mu}$.

The second set of equations comes from a theorem stating a necessary condition on $h_{n}\left(x ; a_{i}\right)$ in order for the function to satisfy the Chebyshev condition.

Theorem 1. Given a function $h_{n}\left(x ; a_{i}\right)$ continuous along with its derivatives in the interval $a \leqq x \leqq b$. Let $x_{1}, \cdots, x_{\mu}$ denote the set of points in $(a, b)$ at which $\left|h_{n}\left(x ; a_{i}\right)\right|$ assumes a maximum. Let the greatest of these maxima be denoted by $M$. Then if $M=$ $M\left(a_{1}, a_{2}, \cdots, a_{n}\right)$ has a minimum value for the parameters specified (i.e., $M=L_{n}$ ), the following set of equations is insoluble for every set of non-zero real numbers $S_{1}, S_{2}$, $\cdots, S_{\mu}$ such that the sign of $S_{k}$ is the same as that of $h_{n}\left(x_{k} ; a_{i}\right), k=1,2, \cdots, \mu$. 


$$
\begin{aligned}
& \left.\frac{\partial h_{n}\left(x_{1} ; a_{i}\right)}{\partial a_{1}} \lambda_{1}+\frac{\partial h_{n}\left(x_{1} ; a_{i}\right)}{\partial a_{2}} \lambda_{2}+\cdots+\frac{\partial h_{n}\left(x_{1} ; a_{i}\right)}{\partial a_{n}} \lambda_{n}=S_{1}\right) \\
& \frac{\partial h_{n}\left(x_{2} ; a_{1}\right)}{\partial a_{1}} \lambda_{1}+\frac{\partial h_{n}\left(x_{2} ; a_{i}\right)}{\partial a_{2}} \lambda_{2}+\cdots+\frac{\partial h_{1}\left(. r_{2} ; a_{2}\right)}{\partial a_{n}} \lambda_{n}=S_{2} \\
& \frac{\partial h_{n}}{\left.\partial x_{\mu} ; a_{i}\right)} \partial \lambda_{1}+\frac{\partial h_{n}\left(x_{\mu} ; a_{i}\right)}{\partial a_{2}} \lambda_{2}+\cdots+\frac{\partial h_{n}\left(x_{\mu} ; a_{i}\right)}{\partial a_{n}} \lambda_{n}=S_{\mu}
\end{aligned}
$$

A theorem similar $t o$ this was proved by Chebyshev (27). The extension to the present form was given by Kircherberger (20).

Example 1. $h_{2}\left(x ; a_{i}\right)=x^{2}+a_{1} x+a_{2},(-1 \leqq x \leqq 1)$.

For this function, equations (1) become:

$$
\begin{gathered}
\left(x^{2}+a_{1} x+a_{2}\right)^{2}-L_{2}^{2}=0, \\
(x-1)(x+1)\left(2 x+a_{1}\right)=0 .
\end{gathered}
$$

Substituting two solutions of (4), $x=+1$ and $x=-1$, into (3) and subtracting the two resulting equations:

$$
a_{1}+a_{1} a_{2}=0 \text {. }
$$

Taking $a_{2}$ equal to -1 , which certainly satisfies (5), the desired polynomial appears to be

$$
x^{2} \pm 2 x-1
$$

with a maximum deviation in the interval $(-1,1)$ of 2 .

Let us use the plus sign in (6). Then the polynomial has a maximum at +1 of +2 and a minimum at -1 of -2 . Equations (2) then become

$$
\begin{aligned}
-\lambda_{1}+\lambda_{2} & =-S_{1} \\
\lambda_{1}+\lambda_{2} & =S_{2}
\end{aligned}
$$

where $S_{1}, S_{2}$ are positive. Thus

$$
\begin{aligned}
& \lambda_{1}=\left(S_{2}+S_{1}\right) / 2, \\
& \lambda_{2}=\left(S_{2}-S_{1}\right) / 2,
\end{aligned}
$$

violating the necessary condition for Chebyshev approximation given by theorem 1 that equations (2) be insoluble. The same would apply if the negative sign had been chosen in (6). Thus (6) is not the desired polynomial.

To solve our problem we note that another solution of (5) is possible, namely, $a_{1}=0$. Substitution of this value for $a_{1}$ gives for $a_{2}$ the value of $-\frac{1}{2}$. Hence the polynomial becomes

$$
x^{2}-\frac{1}{2}
$$


with a maximum deviation of $\frac{1}{2}$ at $x= \pm 1,0$. For this function equations (2) become

$$
\begin{aligned}
-\lambda_{1}+\lambda_{2} & =S_{1}, \\
\lambda_{2} & =-S_{2}, \\
\lambda_{1}+\lambda_{2} & =S_{3},
\end{aligned}
$$

where $S_{1}, S_{2}, S_{3}$ are greater than zero. It is clear that (8) is insoluble in the $\lambda$ 's. Thus the necessary condition that the polynomial approximate zero in the ('hebysher sense is satisfied. That this is the proper polynomial of second degree follows from the fart that the maximum deviations are equal and three in number. This property of polynomials will be shown in section III.

Example 2. $h_{1}\left(x ; a_{1}\right)=x+a_{1} \sin (7 x / 2),(-\pi \leqq x \leqq \pi)$.

Again the problem is to find the value of $a_{1}$ such that the maximum difference of this function from zero in the interval $(-\pi, \pi)$ is a minimum.

Equations (1) becomes

$$
\begin{aligned}
x^{2}+2 a_{1} x \sin (7 x / 2)+a_{1}^{2} \sin ^{2}(7 x / 2)-L_{1}^{2} & =0, \\
(x-\pi)(x+\pi)\left[1+(7 / 2) a_{1} \cos (7 x / 2)\right] & =0 .
\end{aligned}
$$

By a process similar to that used in example 1, it can be shown that the function $x+a_{1}$ $\sin (7 x / 2)$ which satisfies the Chebyshev condition in the interval $(-\pi, \pi)$ is

$$
h_{1}\left(x ; a_{1}\right)=x+0.39 \sin (7 x / 2),
$$

with its maximum deviation equal to 2.75. Its graph is shown in Fig. 1.

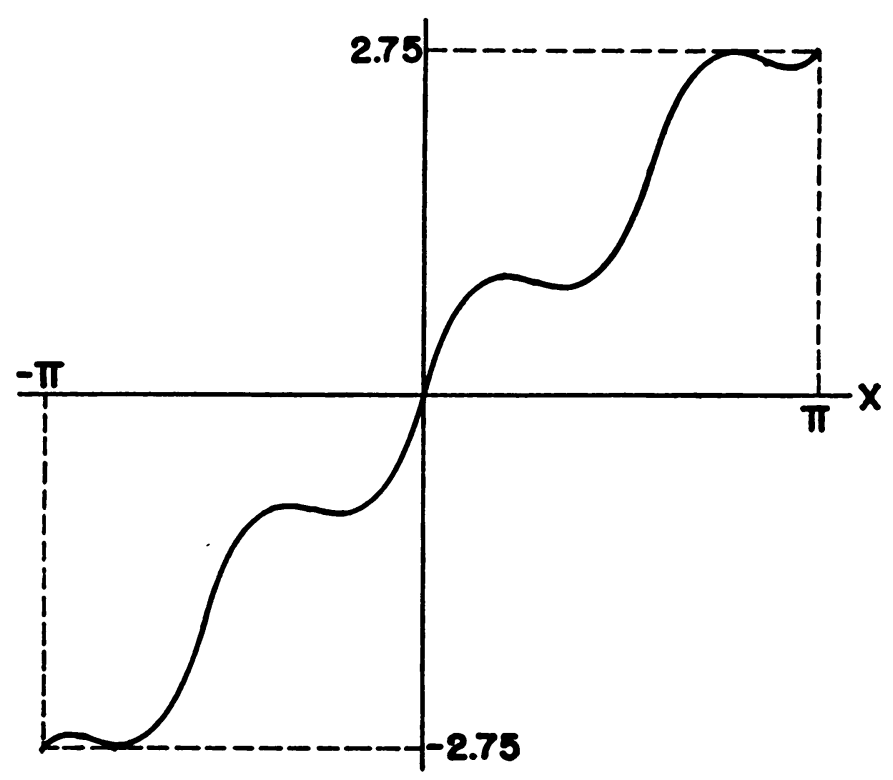

FIG. 1. The function $x+a_{1} \sin (7 x / 2)$ which satisfies the Chebyshev condition in the interval $(-\pi, \pi)$. 
Example 2 was presented primarily as an illustration of a function which satisfies the Chebyshev condition yet whose relative maxima are not all equal. For many general functions, however, the Chebyshev condition is satisfied only when all the relative maxima are equal. This statement is made more precise in the following theorem.

Theorem 2.* Given a function $h_{n}\left(x ; a_{i}\right)$ defined and continuous along with all its derivatives in the interval $(a, b)$. Let $\mu$ be the number of ordinates of $h_{n}\left(x ; a_{i}\right)$ where the magnitude of the function is a relative maximum, including the end points $a$ and $b$ and let $\mu=n+1$, where $n$ is the number of parameters. It is further assumed that either the first derivatives of $h_{n}\left(x ; a_{i}\right)$ with respect to the $a_{i}$ do not vanish at $x_{1}, \cdots, x_{\mu}$ or else if the first derivative vanishes, the first non-vanishing derivative is of odd order. Then if $h_{n}\left(x ; a_{2}\right)$ satisfies the Chebyshev condition in $(a, b)$ the $\mu$ relative maxima are all equal.

This so-called equal maximum property of Chebysher functions will also occur in certain special cases where $\mu \neq n+1$. For instance, in example 2 if the antisymmetrical function had been taken as $x+a_{1} \sin (5 x / 2)$ in the interval $(-\pi, \pi)$, then all four maximum deviations would be equal under the Chebyshev condition, even though there is only one parameter to adjust.

We will now leave the general Chebyshev function and consider the important special case of the Chebyshev polynomials.

3. Chebyshev Polynomials. Given a function $f(x)$ which is finite and continuous along with all its derivatives in the interval $(a, b)$. Let $g_{n}\left(x ; a_{i}\right)=a_{1} x^{n-1}+a_{2} x^{n-2}+$ $\cdots+a_{n}$ be the approximating function. Hence the function which we desire to approximate zero in the Chebyshev sense is

$$
h_{n}\left(x ; a_{i}\right)=f(x)-\left[a_{1} x^{n-1}+a_{2} x^{n-2}+\cdots+a_{n}\right] .
$$

Let $x_{1}, x_{2}, \cdots, x_{\mu}$ be the set of points in the interval $(a, b)$ at which the function given by (11) assumes its maximum deviation $L_{n}$. Under these conditions, equations (2) become

$$
\left.\begin{array}{l}
\lambda_{1} x_{1}^{n-1}+\lambda_{2} x_{1}^{n-2}+\cdots+\lambda_{n-1} x_{1}+\lambda_{n}=S_{1} \\
\lambda_{1} x_{2}^{n-1}+\lambda_{2} x_{2}^{n-2}+\cdots+\lambda_{n-1} x_{2}+\lambda_{n}=S_{2} \\
\cdot \cdot \\
\quad \cdot \\
\lambda_{1} x_{\mu}^{n-1}+\lambda_{2} x_{\mu}^{n-2}+\cdots+\lambda_{n-1} x_{\mu}+\lambda_{n}=S_{\mu}
\end{array}\right\} .
$$

Now let $P(x)$ denote $\lambda_{1} x^{n-1}+\lambda_{2} x^{n-2}+\cdots+\lambda_{n}$, an entire rational function of $(n-1)$ th degree, which by system (12) is determined only in so far as that at a prescribed point it has a prescribed sign. To settle the question of whether equations (12) can be satisfied by a suitable choice of coefficients, it is necessary to consider the number of changes of sign of $S_{1}, S_{2}, \cdots, S_{\mu}$. If there is a change of sign in going from $S_{k}$ to $S_{k+1}$, then there is a root of $P(x)=0$ between $x_{k}$ and $x_{k+1}$. Since we have $(n-1)$ roots at our disposal, and since the number of changes in sign can never exceed the number of roots, then it follows that if the number of sign changes in the $S$ 's is less than or equal to $(n-1)$,

*The statement of this theorem and its proof follows along the lines of one given by LeCorbeiller (22), except that it was found necessary to add a hypothesis to take care of special functions. 
system (12) will be soluble for the $\lambda$ 's. Hence the following proposition has been demonstrated;

A necessary condition for the function $h_{n}\left(x ; a_{i}\right)$ as given by (11) to approximate zero in the Chebyshev sense is that among the set of values $h_{n}\left(x_{1} ; a_{i}\right), h_{n}\left(x_{2} ; a_{i}\right), \cdots, h_{n}$ $\left(x_{\mu} ; a_{i}\right)$ there are at least $n$ changes in sign.

From this it follows that if $h_{n}\left(x ; a_{1}\right)$ as given by (11) is to approximate zero, then equation set (1) will have at least $(n+1)$ common solutions in $(a, b)$, and further the maximum deviation $L_{n}$ will he alternately a maximum and a minimum of the function.

To show that the condition of the non-solubility of equations (2) is not only necessiary but also sufficient for the function given by (11), it will be shown that the condition leads to a unique function $h_{r}\left(x ; a_{i}\right)$.

To prove this, assume that there exists a function $\bar{h}_{n}\left(x ; a_{1}\right)=f(x)-\left(\ddot{a}_{1} x^{n-1}+\bar{a}_{2}\right.$ $x^{n-2}+\cdots+\bar{a}_{n}$ ) which also satisfies the non-solubility condition, in other words, whose maximum deviation is less or equal $L_{n}$. Thus it follow's that $\left(h_{n}-\breve{h}_{n}\right)$ is a polynomial of degree at most $(n-1)$. By the previous discussion there are at least $(n+1)$ points at which $\left|h_{n}\right|=L_{n}$ and at least $(n+1)$ points at which; $\bar{h}_{n} \leqq L_{n}$. Thus $\left(h_{n}-\bar{h}_{n}\right)$ has at least $n$ zero points in $(a, b)$. This means that necessarily $h_{n} \equiv \bar{h}_{n}$.

Let us now take for $f(x)$ the simplest possible form:

$$
f(x)=x^{n} .
$$

Thus the function which we wish to approximate zero may be written as

$$
h_{n}\left(x ; b_{i}\right)=x^{n}+b_{1} x^{n-1}+b_{2} x^{n-2}+\cdots+b_{n}
$$

where the $b$ 's of equation (14) are simply the negatives of the corresponding $a$ 's of equation (11).

For convenience, let us repeat equations (1).

$$
\begin{aligned}
h_{n}^{2}\left(x ; b_{i}\right)-L_{n}^{2} & =0, \\
(x-a)(x-b) h_{n}^{\prime}\left(x ; b_{i}\right) & =0 .
\end{aligned}
$$

Since equation $(1 b)$ is of degree $(n+1)$, it follows from the previous discussion that all its roots must be distinct, and furthermore they must all also be roots of equation (1a). Now since the roots of $h_{n}^{\prime}\left(x ; b_{i}\right)=0$ are roots of $(1 a)$, it follows that there are $(n-1)$ double roots of $(1 a)$. Hence the system of equations

$$
\begin{aligned}
h_{n}^{2}\left(x ; b_{i}\right)-L_{n}^{2} & =0 \\
{\left[h_{n}^{\prime}\left(x ; b_{i}\right)\right]^{2} } & =0
\end{aligned}
$$

will have $(2 n-2)$ common solutions in the form of pairs of double roots. The other two common solutions to $(1 a)$ and $(1 b)$ must occur at $x=a$ and $x=b$. Thus the equations

$$
\begin{aligned}
h_{n}^{2}\left(x ; b_{i}\right)-L_{n}^{2} & =0 \\
(x-a)(x-b)\left[h_{n}^{\prime}\left(x ; b_{i}\right)\right]^{2} & =0
\end{aligned}
$$

will have $2 n$ common solutions. When it is recognized that the coefficient of $x^{2 n}$ in the first of these equations is unity, while in the second it is $n^{2}$, it is seen that the differential 
equation to be solved is

$$
h_{n}^{2}-L_{n}^{2}=\frac{(x-a)(x-b)}{n^{2}}\left(\frac{d h_{n}}{d x}\right)^{2} .
$$

There are four solutions to equation (15) which result from the four possible combinations of signs appearing after the square root of both sides has been taken. These solutions are

$$
h_{n}=L_{n} \begin{aligned}
& \sin \\
& \cos
\end{aligned}\left\{n \operatorname{arc}\left\{\begin{array}{l}
\sin \\
\cos
\end{array}\right\}\left(\begin{array}{c}
-2 x+a+b \\
a-b
\end{array}\right)+C\right\}
$$

where $C$ is the constant of integration. Since our problem is to determine the polynomial of $n$th degree which approximates zero best, we are restricted to the solution

$$
h_{n}=L_{n} \cos \left[n \arccos \left(\frac{-2 x+a+b}{a-b}\right)+C\right] \text {. }
$$

The constant of integration is determined by the requirement that at the boundaries of the interval $(a, b)$, the polynomial must assume its maximum deviation. This requirement is satisfied for $C=0$, though, of course, placing $C$ equal to an integral multiple of $\pi$ would not change the function $h_{n}$.

In order to get (17) in a form which shows its polynomial character more readily, let

$$
z=\arccos \left(\frac{-2 x+a+b}{a-b}\right) \text {. }
$$

Then

$$
\begin{aligned}
& h_{n}=L_{n} \cos n z=L_{n} / 2\left[(\cos z+i \sin z)^{n}+(\cos z-i \sin z)^{n}\right] \\
& =\frac{L_{n}}{2(a-b)^{n}}\left[\left(-2 x+a+b+\sqrt{4 x^{2}-2(a+b) x+4 a b}\right)^{n}\right. \\
& \quad+\left(-2 x+a+b-\sqrt{\left.4 x^{2}-2(a+b) x+4 a b\right)^{n}}\right] .
\end{aligned}
$$

For the coefficient of $x^{n}$ to be unity, as given in (14),

$$
L_{n}= \pm \frac{(a-b)^{n}}{2^{2 n-1}} .
$$

Tabulation of (hebyshev polynomials in the interval $(a, b)$.

From equation (18) the following set of polynomials are obtained;

$$
\begin{aligned}
& h_{1}=x-\frac{a+b}{2} \\
& h_{2}=x^{2}-\frac{3(a+b)}{4} x+\frac{a^{2}+6 a b+b^{2}}{8} \\
& h_{3}=x^{3}-\frac{9(a+b)}{8} x^{2}+\frac{3 a^{2}+12 a b+3 b^{2}}{8} x-\frac{a+b}{32}\left(a^{2}+14 a b+b^{2}\right)
\end{aligned}
$$


It is usually convenient to deal with the Chebyshev polynomials in the interval $(-1,1)$ instead of the interval $(a, b)$. From (17) and (19), these functions may compactly be written as

$$
\frac{1}{2^{n-1}} \cos (n \operatorname{arc} \cos x) \text {. }
$$

For our purposes the polynomials of unit maximum amplitude in the interval $(-1,1)$ will be more useful: i.e., the polynomials given by

$$
T_{n}^{\prime}(x)=\cos (n \operatorname{arc} \cos x) .
$$

Hereafter, referenees to Chebyshev polynomials will means those functions given by (20). The first ten such functions are tabulated in Table I for convenience. Fig. 2 shows a few of these polynomials.

Table I. Tabelation of the Chebyshev Polynomials

$$
T_{n}(x)=\cos (n \arccos x)
$$

$$
\begin{aligned}
& T_{0}(x)=1 \\
& T_{1}(x)=x \\
& T_{2}(x)=2 x^{2}-1 \\
& T_{3}(x)=4 x^{3}-3 x \\
& T_{4}(x)=8 x^{4}-8 x^{2}+1 \\
& T_{5}(x)=16 x^{5}-20 x^{3}+5 x \\
& T_{6}(x)=32 x^{6}-48 x^{4}+18 x^{2}-1 \\
& T_{7}(x)=64 x^{7}-112 x^{5}+56 x^{3}-7 x \\
& T_{8}(x)=128 x^{8}-256 x^{6}+160 x^{4}-32 x^{2}+1 \\
& T_{9}(x)=256 x^{9}-576 x^{7}+432 x^{5}-120 x^{3}+9 x \\
& T_{10}(x)=512 x^{10}-1280 x^{8}+1120 x^{6}-400 x^{4}+50 x^{2}-1
\end{aligned}
$$

Another convenient form for $T_{n}(x)$ is, from (18),

$$
T_{n}(x)=\frac{1}{2}\left[\left(x+\sqrt{\left.x^{2}-1\right)^{n}}+\left(x-\sqrt{x^{2}-1}\right)^{n}\right] .\right.
$$

Also, the expansion of (20) gives:

$$
T_{n}(x)=\sum_{\lambda=0}^{\lfloor n / 21}(-1)^{\lambda}\left(\begin{array}{c}
n-\lambda \\
\lambda
\end{array}\right) \frac{n}{n-\lambda} \frac{x^{n-2 \lambda}}{2^{2 \lambda-n+1}} \quad(n \geqq 1)
$$

where $[n / 2]$ denotes the largest integer contained in the set $0, \cdots, n / 2$.

A still further form for $T_{n}(x)$, which may be checked by using the original differential equation, is

$$
T_{n}(x)=2^{n}(-1)^{n} \frac{n !}{(2 n) !} \sqrt{1-x^{2}} \frac{d^{n}}{d x^{n}}\left(1-x^{2}\right)^{n-1 / 2} \quad(n \geqq 0)
$$


Further properties of the Chebyshev polynomials. 1. Recurrence relation. Letting again $z=\operatorname{arc} \cos x, T_{n}=\cos n z$. Since $\cos (n+1) z+\cos (n-1) z=2 \cos n z \cos z$, then $T_{n+1}+T_{n-1}=2 T_{n} T_{1}$. Thus taking into account from (20) the values of $T_{n}$ for $n=0,1$ :

$$
T_{n+1}=2 x T_{n}-T_{n-1}
$$

where

$$
T_{0}=1 \text { and } T_{1}=x
$$

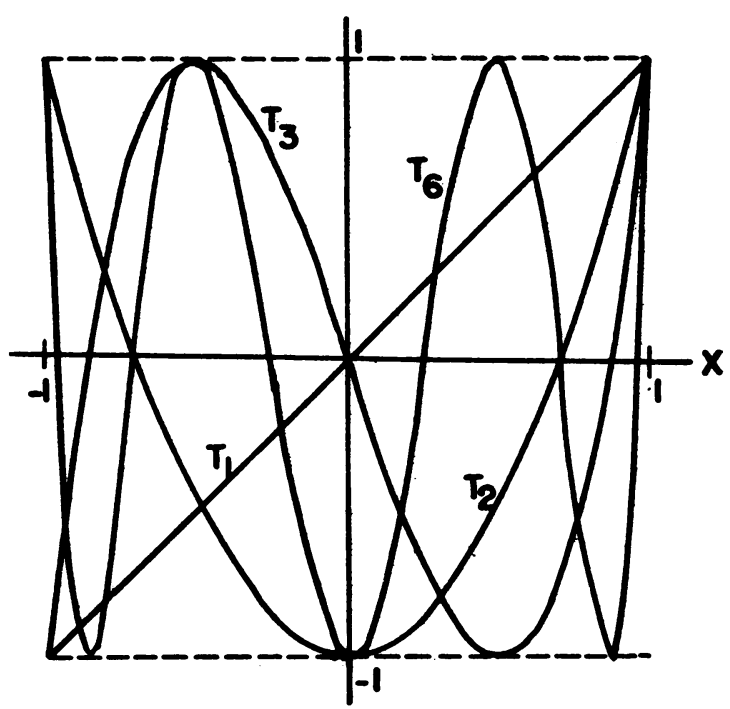

FIG. 2. The Chebyshev polynomials $T_{n}(x)=\cos (n \operatorname{arc} \cos x)$.

2.Orthogonal property. The $T$ 's are othogonal to each other in the interval $(-1,1)$ with respect to the function $1 / \sqrt{1-x^{2}}$, as is easily seen from the fact that

$$
\int_{-1}^{1} T_{n}(x) T_{m}(x) \sqrt{1-x^{2}}=\int_{0}^{\pi} \cos n \theta \cos m \theta d \theta=\left\{\begin{array}{l}
0(m \neq n) \\
\frac{\pi}{2}(m=n \geqq 1) \\
\pi(m=n=0)
\end{array}\right.
$$

3. Generating functions. One generating function which may be used to define the $T_{n}(x)$ is:

$$
\frac{1-x t}{1-2 x t+t^{2}}=\sum_{n=0}^{\infty} T_{n}(x) t^{n} . \quad(t<1)
$$

A second such function is

$$
\ln \frac{1}{\sqrt{t^{2}-2 x t+1}}=\sum_{n=1}^{\infty} T_{n}(x) \frac{t^{n}}{n} . \quad(t<1)
$$

4. Maximization of the largest root. This property will be stated in the form of a 
theorem, which will be useful later on in the application of the Chebyshev polynomials to antennas. The outline of the proof is given in ref. (14).

Theorem 3. Let $C(a)$ be the class of polynomials with real coefficients of degree $n$ having all of their roots in the interval $(-1,1)$ and such that for every polynomial $P(x)$ belonging to this class, $P(x)=(-1)^{n} P(-x), P(1)=1$, and if $x_{0}$ is the largest root of $P(x)$ then $|P(x)| \leqq a$ for $|x| \leqq\left|x_{0}\right|<1$. Then it follows that there exists a polynomial $M(x)$ in $C(a)$ which maximizes $\left|x_{0}\right|$. Furthermore, $M(x)$ assumes the maximum value of $\pm a$ at $(n-1)$ points within the region $|x| \leqq\left|x_{0}\right|$, and $M(x)=a T_{n}\left(z_{0} x\right)$ where $z_{0}$ satisfies the relations: $T_{n}\left(z_{0}\right)=1 / a ; z_{0}>\cos (\pi / 2 n)$.

5. The $U_{n}(x), V_{n}(x)$, and $W_{n}(x)$ functions. Whether one considers the nonlinear differential equation (see eq. 15)

$$
\left(\frac{d y}{d x}\right)^{2}+\frac{n^{2}}{1-x^{2}}\left(y^{2}-1\right)=0
$$

or the linear differential equation

$$
\left(1-x^{2}\right) \frac{d^{2} y}{d x^{2}}-x \frac{d y}{d x}+n^{2} y=0
$$

both of which are satisfied by $T_{n}(x)=\cos (n \operatorname{arc} \cos x)$, it is apparent that there are three other solutions, which may be written

$$
\begin{aligned}
U_{n}(x) & =\sin (n \arccos x), \\
V_{n}(x) & =\cos (n \arcsin x), \\
W_{n}(x) & =\sin (n \arcsin x),
\end{aligned}
$$

one of which may be taken with $T_{n}(x)$ as the second independent solution, from which the other two solutions may be derived. If $U_{n}(x)$ is taken as the second solution, it can easily be shown that

$$
\begin{aligned}
V_{2 n}(x) & =(-1)^{n} T_{2 n}(x), \\
V_{2 n+1}(x) & =(-1)^{n} U_{2 n+1}(x), \\
W_{2 n}(x) & =(-1)^{n} U_{2 n}(x), \\
W_{2 n+1}(x) & =(-1)^{n} T_{2 n+1}(x) .
\end{aligned}
$$

Table II. Tabulation of the Functions

$$
\begin{aligned}
& U_{n}(x)=\sin (n \operatorname{arc} \cos x) \\
U_{0}(x)= & 0 \\
U_{1}(x)= & \sqrt{1-x^{2}} \\
U_{2}(x)= & x \sqrt{1-x^{2}} \\
U_{3}(x)= & \left(4 x^{2}-1\right) \sqrt{1-x^{2}} \\
U_{4}(x)= & \left(8 x^{3}-4 x\right) \sqrt{1-x^{2}} \\
U_{5}(x)= & \left(16 x^{4}-12 x^{2}+1\right) \sqrt{1-x^{2}}
\end{aligned}
$$


The $U_{n}$ functions also satisfy the equal maximum and minimum property throughout the interval $(-1,1)$ that the $T_{n}$ polynomials do. The former functions are irrational, however, as Table II shows, and have $n$ extremal points, rather than $(n+1)$, as fig. 3 shows.

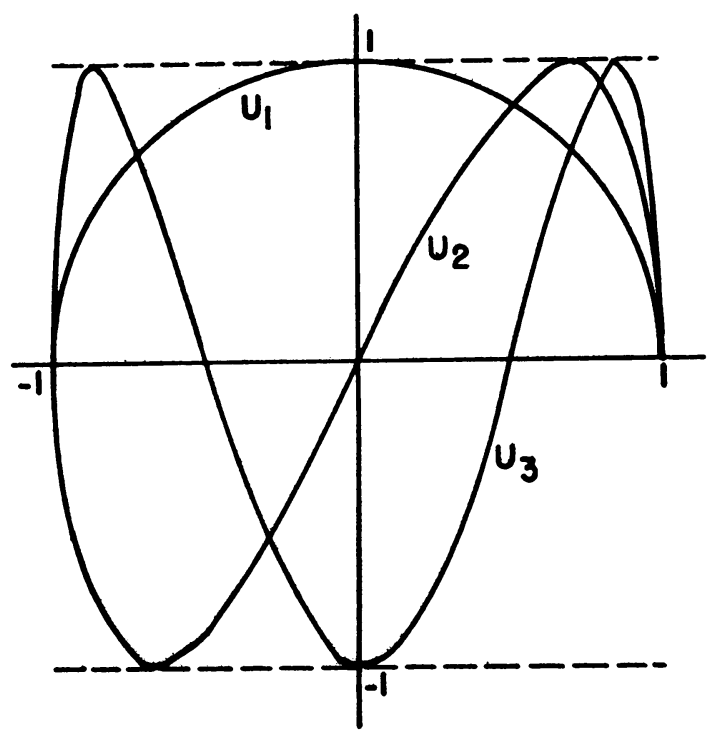

Frg. 3. The functions $U_{n}(x)=\sin (n \operatorname{arc} \cos x)$.

6. Relation of the $T_{n}$ polynomials to the Bessel functions $J_{n}(t)$. The Bessel function $J_{n}(t)$ in the time domain will produce, through the Fourier integral, the following function in the frequency domain:

$$
F\left[J_{n}(t)\right] \equiv \int_{-\infty}^{\infty} J_{n}(t) e^{-i \omega t} d t= \begin{cases}2(-i)^{n} \frac{T_{n}(\omega)}{\sqrt{1-\omega^{2}}} & \omega^{2}<1 \\ 0 & \omega^{2}>1\end{cases}
$$

Hence, we may write

$$
J_{n}(t)=\frac{(-i)^{n}}{\pi} \int_{-1}^{1} e^{i \omega t} \frac{T_{n}(\omega)}{\sqrt{1-\omega^{2}}} d w .
$$

7. Relation to the Neumann polynomials. The Neumann polynomials $0_{n}(z)$ are defined by the relation

$$
0_{n}(z)=\frac{1}{2} \int_{0}^{\infty}\left[\left(x+\sqrt{1+x^{2}}\right)^{n}+\left(x-\sqrt{1+x^{2}}\right)^{n}\right] e^{-x z} d x .
$$

Hence this relation can be written

$$
0_{n}(z)=(-i)^{n} \int_{0}^{\infty} e^{-x z} T_{n}(i x) d x .
$$

8. Relation of $T_{n}$ and $U_{n}$ with Lissajous figures. Let a closed cosine curve with $n$ periods be drawn on the surface of a cylinder with unit radius and with the axis in the 
$z$ direction, as shown in Fig. 4. Thus we mav say that the curve is given in space by $z=$ $\cos n \theta$ and $\mathrm{r}=1$. Therefore, the abscissa of the orthogonal projection of this curve on the plane formed by the $z$-axis and the $\theta=0$ line is then $x=\cos \theta$. Hence the projected

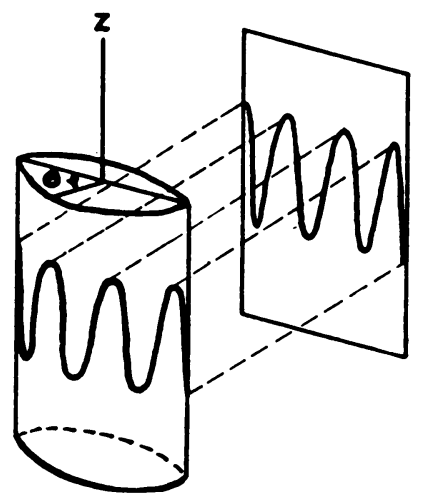

Fia. 4. The curve $\cos 7 \theta$ on a cylinder of unit radius and its projection on a plane to form a Lissajous pattern.

figure gives the relation between $x=\cos \theta$ and $z=\cos n \theta$ and is thus represented by

$$
z=\cos (n \operatorname{arc} \cos x) .
$$

If time is introduced as the variable instead of $\theta$, the projection of the curve $z=\cos$ $n \theta$ can be written in the parametric form

$$
\begin{aligned}
& z=\cos n t, \\
& x=\cos t,
\end{aligned}
$$

and thus represent Lissajous figures for an integral ratio of the two composing frequencies.

If the curve had been taken as $z=\sin n \theta$, then the projections would have been proportional to the $U_{n}(x)$ functions instead of to the $T_{n}(x)$.

9. $T_{n}(x)$ and $U_{n}(x)$ in relation to Fourier series. A similar geometric interpretation can be given (30) to show the relation of the $T_{n}$ and $U_{n}$ functions to Fourier series. The connection can be seen, however, quite easily in the following way. Let $Y(\theta)$ be a given function in an interval, say $(a, b)$. Developing this function in a Fourier series, we have

$$
Y(\theta)=\sum_{n=0}^{\infty}\left(a_{n} \cos n \theta+b_{n} \sin n \theta\right) . \quad(a \leqq \theta \leqq b)
$$

If $x$ is set equal to $\cos \theta$ :

$$
Y\left(\cos ^{-1} x\right)=\sum_{n=0}^{\infty}\left[a_{n} T_{n}(x)+b_{n} U_{n}(x)\right] . \quad\left(a \leqq \cos ^{-1} x \leqq b\right)
$$

10. Expansion of an arbitrary function in Chebyshev polynomials. By taking into account the orthogonality property, it is an easy matter to show that if the series

$$
2 c_{0}+\sum_{n=1}^{\infty} c_{n} T_{n}(x)
$$


converges uniformly to the sum $f(x)$ in the interval $-1 \leqq x \leqq 1$, then

$$
c_{n}=\frac{2}{\pi} \int_{-1}^{1} f(x) T_{n}(x) \frac{d x}{\sqrt{1-x^{2}}} .
$$

As an example of the use of a function expanded in Chebyshev polynomials, consider the curve of plate current versus grid voltage for a triode. (31) Linear theory of triode amplification is based on the assumption that the operating region is so small that the characteristic may be replaced by a straight line throughout the region with negligible error. This involves considering only the linear term in the power series expansion about the operating point. To calculate the harmonic distortion resulting from a finite grid swing, the higher derivative terms in the power series become important. From experimental measurement the coefficients of the Taylor series can only be derived from the limit towards which the measurements converge for an infinitely small grid swing.

Consider a sine wave applied to the grid, so that the grid voltage $e_{0}(t)$ is given by

$$
e_{o}(t)=e_{00}+a \cos \omega t \text {. }
$$

The application of $e_{\theta}(t)$ causes a plate current to flow of the form

$$
i_{a}(t)=I_{0}+I_{1} \cos \omega t+I_{2} \cos 2 \omega t+\cdots \text {. }
$$

Now if we write

then

$$
x=\cos \omega t
$$

$$
i_{a}(t)=I_{0}+I_{1} T_{1}(x)+I_{2} T_{2}(x)+\cdots \cdot
$$

In other words, the amplitudes of the higher harmonics which are measured experimentally, are, for a given operating point and grid swing, the coefficients of the triode characteristic expanded in a series of Chebyshev polynomials. Thus the expression of the triode characteristic in this form is easy to obtain experimentally, and when once !written, gives the information regarding the amount of harmonics under particular operating conditions.

Application of Chebyshev Polynomials to Antennas. It is frequently the problem in antenna design that the beam should be as narrow as possible, the power gain should be a maximum, and the sidelobes should be relatively small. These requirements are normally not easy or even possible to satisfy simultaneously, so the problem arises as to what to term the optimum field pattern. For this discussion we shall term that field pattern optimum which has a minimum beam width for a specified sidelobe level.

To determine a method for obtaining the optimum pattern, consider an array of $n$ isotropic point sources of uniform spacing and all in the same phase, as shown in fig. 5 . For even $n$, the array is given by $5 a$; for odd $n$, by $5 b$. The amplitudes of the individual sources are denoted by the $A$ 's as shown. Hence the total field for $5 a$ in a direction $\theta$ is given, for large distances, by the expression (21)

$$
E_{\text {coen }}=2 A_{1} \cos \phi / 2+2 A_{2} \cos 3 \phi / 2+\cdots+2 A_{n / 2} \cos (n-1) \phi / 2,
$$

and for $5 b$,

$$
E_{\text {odd }}=2 A_{0}+2 A_{1} \cos \phi+2 A_{2} \cos 2 \phi+\cdots+2 A_{(n-1) / 2} \cos (n-1) \phi / 2,
$$

where $\phi=2 \pi d / \lambda \sin \theta$ and $d$ is the distance between point sources. It is clear that (34) 

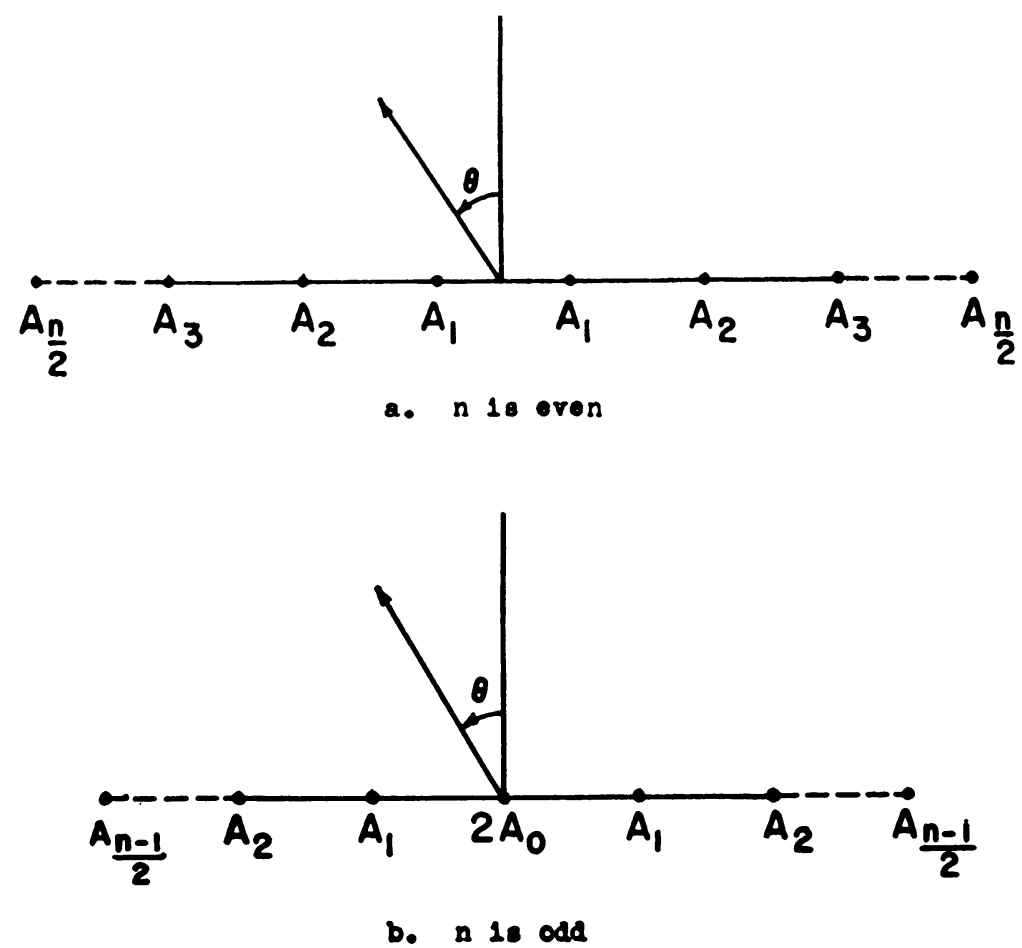

Fig. 5. Linear broadside array of $n$ isotropic sources with uniform spacing. a. $n$ is even. b. $n$ is odd.

and (35) may be written in the form:

$$
\begin{aligned}
& E_{\text {evon }}=2 \sum_{k=1}^{n / 2} A_{k} \cos (2 k-1) \phi / 2=2 \sum_{k=1}^{n / 2} A_{k} T_{2 k-1}(x), \\
& E_{\text {odd }}=2 \sum_{k=0}^{(n-1) / 2} A_{k} \cos k \phi=2 \sum_{k=0}^{(n-1) / 2} A_{k} T_{2 k}(x),
\end{aligned}
$$

where $x=\cos \phi / 2$.

It is now the problem to determine the value of the $A$ 's in (36) and (37) so that the optimum pattern is obtained. One way is to make all of the $A$ 's equal. This, indeed, gives a maximum gain and a narrow beam width, but the level of the side lobes is very high. Another way is to make the $A$ 's proportional to the binomial coefficients, in which case the side lobes disappear altogether if the spacing between elements is less than half a wave length, but unfortunately increased beam width and loss of gain result.

Now let us consider Dolph's method (14) of using the Chebyshev polynomials to obtain an optimum pattern. Suppose we have an array of $n$ sources as in Fig. 5, where $n$ may be odd or even. Let the specified ratio of the main lobe maximum to the minor lobe level be $R$. The problem is to find the relative source strengths for the pattern to be optimum in our prescribed sense.

The forms (36) and (37) show that the field due to the $n$ given sources is described by a polynomial of $(n-1)$ th degree, which may be denoted by $P_{n-1}(x)$. The solution of the equation $P_{n-1}(x)=R$ will give $x_{R}$, the point at which the pattern assumes its. 
maximum value. Letting $y=x x_{k}$, then $(1 / R) P_{n-1}(y)$ will have a value of unity at $y=1$, and we have that $y$ (rather than $x$ ) is equal to $\cos \phi / 2$. It is necessary that $P_{n-1}(y)$ have all its roots in the interval $(-1,1)$, and furthermore be an even function about the origin. Thus $P_{n-1}(y)$ satisfies the requirements of Theorem 3 . Hence it follows that if the distance from the greatest zero to the point $y=1$ is to be a minimum (which must be the case for the beam width to be a minimum), then $P_{n-1}(y)$ must be proportional to the ('hobyshev polynomial $T_{n-1}(x)$. Fxamples appear in Dolph 's paper.

Relation of the Chebysher polynomials to filters. ('onsider the network shown in fig. 6 ,

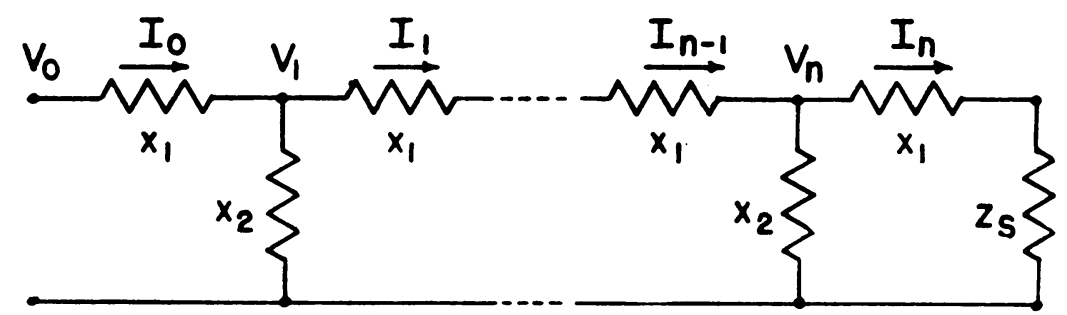

Fig. 6. Finite chain of reactive elements connected in ladder form.

which is composed of $n$ identical circuits of isolated lossless elements. Let the driving emf at the input terminals be a sinusoidal source of amplitude $V_{0}$ and of angular frzquency $\omega$. Let $x_{1}$ and $x_{2}$ represent the reactances of the circuit elements at this frequency. Then

$$
x_{1} I_{k}=x_{2}\left(I_{k-1}+I_{k+1}-2 I_{k}\right)
$$

or, by placing $x=x_{1} / x_{2}+2$,

$$
I_{k+1}-x I_{k}+I_{k-1}=0 .
$$

From equation (38) it can easily be shown that if

$$
y_{n}(x)=x^{n}-\left(\begin{array}{c}
n-1 \\
1
\end{array}\right) x^{n-2}+\left(\begin{array}{c}
n-2 \\
2
\end{array}\right) x^{n-4}-\cdots
$$

then in terms of $I_{0}$ an $I_{1}, I_{k+1}$ may be expressed as

$$
I_{k+1}=y_{k} I_{1}-y_{k-1} I_{0} \text {. }
$$

In terms of $I_{0}$ and $V_{0}$ :

$$
\begin{aligned}
& V_{k}=-y_{k-1} x_{1} I_{0}+\left(y_{k-1}-y_{k-2}\right) V_{0}, \\
& I_{k}=\left(y_{k}-y_{k-1}\right) I_{0}-y_{k-1} V_{0} / x_{2} .
\end{aligned}
$$

The $y$ 's are polynomials satisfying the same recurrence relation as (38). By comparing the expansion of $y_{n}(x)$ with the expansion (22), the following relation between the $y$ 's and the Chebyshev polynomials can be derived:

$$
y_{n}(x)=2\left[T_{n}(x / 2)+T_{n-2}(x / 2)+\cdots+T_{1}(x / 2) \text { if } n \text { is odd. }\right]
$$


Through the use of equation (40) not only can the successive currents and voltages in the network shown in fig. 6 be expressed in terms of Chebysher polynomials, but also the input impedance and transfer impedance for any load $z_{s}$.

Application to directional couplers. It has been pointed out by $\mathrm{F}$. Bolinder (2) that if one wishes to maximize the ratio of the width of the pass band to the level of the reverse wave in the pass band in a directional coupler, then for a given number of elements one will choose the coupling factors in such a way that the rariation of the reversed current level with distance will be a Chebysher polynomial.

Conclusion. This paper has presented the properties of the Chebysher approximation method and the Chebyshev polynomials which may be of interest and use to engneers and physicists. The application of the method to filter design has not been corered here because of the length of the subject and the detail required. Glowatzki (16) gives tables for determining the number and sizes of elements needed in a filter which meets certain specified requirements. A number of other referenc's on this subject are given in the bibliography, where also may be found references to articles of a detailed mathematical nature for those interested in this aspect of the problem:

\section{Bibliography}

[1] E. Borel, Lecons sur les fonctions de variables, rceles et les developpements en series de poi!ynomes, Gauthier-Villars, Paris, 1905.

[2] F. Bolinder, Approximate theory of the directional coupler, Proc. IRE, 39, 1951, 291.

[3] W. Cauer, L'eber die Variabeln eines passiven Vierpols, Sitzungsberichte der Preuss. Akad. der Wiss., 1927.

[4] W. Cauer, Vierpole, E.N.T., 1929.

[5] W. Cauer, Siebschaltungen, V.D.I., Verlag G.M.B.H. Berlin, 1931. (Condensed version in Physics, 2, No. 4, 1932, 242-268.)

[6] W. Cauer, Frequenzweichen konstanten Betriebswiderstandes, E.N.T., Bd. 16, 1939, 96-120.

[7] J. Chokhatte, Sur quelques proprietes des polynomes de Tchebycheff, Comptes Rendus, 166, 1918, 28-31.

[8] J. Chokhatte, On a general formula in the theory of Tchebycheff polynomials and its applications, Trans. Amer. Math. Soc., 29, 1927, 569-583.

[9] J. Chokhatte, Sur une formule general dans la theorie des polynomes de Tchebycheff et ses applications, Comptes Rendus, 181, 1925, 329-331.

[10] J. Chokhatte, Sur quelques applications des polynomes de Tchebycheff a plusieurs variables, Comptes Rendus, 183, 1926, 442-444.

[11] J. Chokhatte, Sur le developpement de l'integrale $\int_{a}^{b} \frac{p(y)}{x-y} d y$ en fraction continue et sur les polynomes de Tchebycheff, Rendiconti del Circolo Matematico di Palermo, 47, 1923, 25-46.

[12] A. Colombani, La theorie des filtres electriques et les polynomes de Tchebichef, J. de. Physique et le Radium, 7, No. 8, 1946, 231-243.

[13] P. Coulombe, Calcul rationnel des filtres en echelle, Bull. Soc. Fran. des Elec., 6, 1946, 103-110.

[14] C. L. Dolph, A current distribution for broadside arrays which optimizes the relationship between beam width and side-lobe level, Proc. IRE, 34, 1946, 335-348.

[15] R. Feldtkeller, Einfuhrung in die Theorie der Rundfunksiebschaltungen, 3, Auf., S. Hirzel, Leipzig, 1945.

[16] E. Glowatzki, Entwurf und Beispiele symmetrischer Siebschaltungen nach der Methode von W. Cauer, E.N.T., Teil I, Heft 9; Teil II, Heft 10; 1933.

[17] E. A. Guillemin, A recent contribution to the design of electric filter networks, Jnl. Math. and Phys., 11, $1932,150-211$.

[18] E. S. Guillemin, Communication networks, II, John Wiley and Sons, New York, 1935.

[19] R. Julia, Sur la theorie des filtres de W. Cauer, Bull. Soc. Fran. des Elec., 5, 1935, 983-1066.

[20] P. Kircherberger, Uber Tchebychefsche Annaherungsmethoden, Inaugural dissertation, Gottingen, 1932.

[21] J. D. Kraus, Antennas, McGraw-Hill, New York, 1950, 97-110. 
[22] P. Le Corbeiller, IIcthode d'approximation de Tchebychef et application aux filtres de frequences, RGE, 40, 1936, 651-657.

[23] F. Leja, Sur less polynomes de T'chebycheff et la fonction de Green, Ann. Soc. Polon, Math., 19, 1946, 1-6.

[24] Stephan Lipkit, C'ber die Anzahl der Nullstellen von Tschebyscheff Polynomen, Monatsh. Math. Phys., 51, 1944, 173-178.

[25] R. L. Pritchard, Optimum directivity patterns jor linear arrays, Tech. Memorandum \#7, Acoustics Research Labs., Harvard, 19.50, 3-5. Alsn. Directivity of linear point arra!!s, Ph.D. thesis, Harvard Liniversity.

[26] P. L. Tchebycheff, Theorie des mecanismes' connus sous: le nom de parallelogrammes, ()euvres, 111-143.

$[27 \mid$ P. I. Tchebycheff, Sur les questions de minima qui se rattachent a la representation approximatives des fonctions, Mem. Acad. Imperiale des Sci. ile Sit. Petersbourg, t. VII, 1859, 199-291. Also, Oeuvres, t. I, 273-378.

[2S] P. L. Tehebycheff, Sur les fonctions qui different le moins possible de zero, Jnl. des Mathematiques pures et appliquees, t. XIX, 1874, 3I!)-3Hti. ()euvres, t. II, 189-215.

[2(1) P. L. Tchebycheff, Sur les fonctions tui securtent peu de zero pour certaines valeurs de la cariable, Oeuvres, t. II, 335-356.

[30] B. van der Pol and Th. J. Weijers, T'chelygcheff polynomials and their relation to circular functions, Bessel functions, and Lissajous figures, Physicu, 1933, 1, 78-96.

[31] B. van der Pol and Th. J. Weijers, Fine structure of triole characteristics, Physica, 1, 1934, 481-496. 